SCHWERPUNKT

\section{Forschungsperspektiven für Postwachstum in Technik, Wirtschaft und Gesellschaft}

\section{Einleitung in den Schwerpunkt}

\author{
von Jürgen Kopfmüller und Linda Nierling, \\ Institut für Technikfolgenabschätzung \\ und Systemanalyse, Karlsruhe, und André \\ Reichel, Karlshochschule International \\ University, Karlsruhe
}

„Nieder mit Nachhaltiger Entwicklung!“ rief Serge Latouche 2002 den Teilnehmenden einer UNESCO-Konferenz in Paris $2002 \mathrm{zu}$ - und fügte hinzu: „Vive la décroissance conviviale!“, was sich in Anlehnung an den vorherrschenden deutschen Sprachgebrauch frei übersetzen lässt mit: „Es lebe die konviviale Postwachstumsökonomie!“ Darin kommt zum Ausdruck, dass der Postwachstumsbegriff oder „Décroissance“ (eigentlich „Schrumpfung“ oder „Wachstumsrücknahme") von Beginn an dem im Forschungsmainstream mittlerweile geläufigen Begriff einer Nachhaltigen Entwicklung entgegengesetzt wurde. Nachhaltige Entwicklung wird aus einer Postwachstumsperspektive häufig als Leitbild gesehen, das eine Weiterführung des wirtschaftlichen Wachstumskurses unterstütze bzw. nicht verhindere und dem es bis heute nicht gelungen sei, den Widerspruch zwischen Wirtschaftsleistung und Umweltverbrauch aufzulösen und auf eine grundlegende Entkopplung zwischen diesen beiden Faktoren hinzuwirken.

Allerdings ist mit „Décroissance“ nicht einfach ein Wiederaufleben der Wachstumskritik der 1970er Jahre gemeint. Von Beginn hat sich der Postwachstumsdiskurs explizit sozialen Fragen der gerechten Verteilung, von demokratischer Teilhabe und der Kritik am Kapitalismus gewidmet und von dieser Warte aus ein politisches Transformationsprogramm gefordert und in Ansätzen formuliert. Dessen Eckpunkte sind eine zumin- dest teilweise Abkehr vom globalen Kapitalismus und den damit verbundenen globalen Lieferketten sowie entsprechenden Abhängigkeitsstrukturen, insbesondere zwischen armen und reichen Staaten, eine Wiederentdeckung und Stärkung lokaler Wirtschaftskreisläufe, eine basisdemokratische Wende durch kooperative und gemeinwohlorientierte Formen des Wirtschaftens, eine radikale Kritik bestehender Konsum- und Produktionsmuster, die Hinwendung zu suffizienten Lebensstilen, eine Neubewertung von Rolle und Umfang der Erwerbsarbeit sowie eine stärkere Rolle des Staates bei der (Re-)Regulierung der Wirtschaft und der Produktion öffentlicher Güter bzw. der verschiedenen Bereiche der Daseinsvorsorge.

In der bisherigen Diskussion kann zwischen einem engeren und einem weiteren Postwachstumsbegriff unterschieden werden. Der engere Postwachstumsbegriff lässt sich am ehesten mit den Begriffen „Décroissance“ und „Degrowth“" übersetzen und meint das Programm, welches in der Folge der Arbeiten von Autoren wie Serge Latouche, Tim Jackson oder Niko Paech entstanden ist und die oben angeführten Ideen enthält (Latouche 2004; Jackson 2009; Paech 2009; Latouche 2010). Der breitere Postwachstumsbegriff wird vor allem im Englischen unter „Postgrowth“ geführt und umfasst all jene Perspektiven, die sich in differenzierterer Weise mit dem Wachstumsbegriff angesichts absoluter ökologischer Belastungsgrenzen befassen, gutes Wachstum und Lebensqualität von ,unökonomischem“ Wachstum unterscheiden (Hermann Daly), menschenwürdiges Leben und seine Rahmenbedingungen in den Mittelpunkt stellen und das „Ende des Wachstums" (Richard Heinberg) als in zunehmendem Maße empirisches Phänomen und als zentrale gesellschaftliche Herausforderung betrachten (Daly/Farley 2003; Speth 2009; Heinberg 2011).

Neben dem als schwierig charakterisierbaren Verhältnis des Postwachstums-Diskurses zum Nachhaltigkeitsleitbild besteht Klärungswie auch Forschungsbedarf ebenso in der Frage, welche Rolle Technologien für Postwachstum spielen bzw. spielen könnten und wie genau Technologien in bestimmten Bereichen aussehen, die mit Postwachstumsansätzen vereinbar wären. Beide Perspektiven werden in diesem Schwerpunkt, neben anderen, adressiert. 


\section{Zu den Beiträgen}

Dieser Themenschwerpunkt ist in der Folge eines Workshops entstanden, der gemeinsam von ITAS und der Karlsruher Karlshochschule International University im November 2015 durchgeführt wurde. Ziel der Veranstaltung war es, sowohl mit erfahrenen als auch jüngeren WissenschaftlerInnen neue Forschungsperspektiven im Themenfeld einer Postwachstumsgesellschaft zu diskutieren. Es wurden dabei vier thematische Schwerpunkte als Zugang gewählt: Konzeptionen von Postwachstum, Technologie, Stadt und Unternehmen.

In diesem Schwerpunkt werden wesentliche Ergebnisse und Erkenntnisse des Workshops dokumentiert und reflektiert. Hierbei bleiben die vier thematischen Schwerpunkte bestehen. Der erste Beitrag von Stephan Lorenz systematisiert die oftmals heterogene Begriffs- und Akteurslandschaft, die hinter den Begriffen „Postwachstum" und „Degrowth" steht und bietet damit eine theoretisch-konzeptionelle Reflexionsgrundlage für den aktuellen Diskurs an. Im Anschluss entwickelt Barbara Muraca auf Grundlage der theoretischen Ansätze von Nicholas GeorgescuRoegen und Ivan Illich einen Vorschlag zum konzeptionellen Umgang mit Technik in der Postwachstumsdebatte, in dem sie „Viabilität" und „Konvivialität" als Leitbegriffe identifiziert. Diese können als normativer Rahmen angesehen werden, der als Orientierung für die Entwicklung und Bewertung von Technik in einer Postwachstumsgesellschaft dienen kann. Im darauf folgenden Beitrag stellen Colette Waitz und Sarah Meyer-Soylu die Potenziale von Reparaturcafés für eine Stadtentwicklung im Sinne von Postwachstumsideen auf der Basis eigener empirischer Erhebungen dar. Es zeigt sich, dass Reparaturcafés durchaus ein positives Transformationspotenzial in Richtung Postwachstum haben und auf individueller Ebene erforderliche Veränderungen hervorrufen können. Dirk Posse beschreibt in seinem Beitrag den Kontext, in dem sich Unternehmen in der Postwachstumsperspektive bewegen und geht der - auf den ersten Blick paradoxen Frage nach, welche Motivationen Unternehmen haben, die eine Strategie verfolgen, ohne Wachstumsorientierung zu wirtschaften, und wie dies realisiert werden kann. Er beschreibt Faktoren, die Unternehmen nicht nur als Treiber, sondern auch als Getriebene der Wachstumsideologie erscheinen lassen und zeigt Beispiele für Handlungsspielräume in Richtung Geschäftsmodelle für Postwachstum auf.

Die Frage nach neuen Forschungsperspektiven für eine Postwachstumsgesellschaft beinhaltet neben inhaltlichen Aspekten auch den Blick auf institutionelle Gegebenheiten und Voraussetzungen. Diese Perspektive nimmt Steffi Ober anhand einer Betrachtung der gegenwärtigen Förderpolitik in Deutschland ein, indem sie Möglichkeiten aber auch Barrieren für eine thematische Orientierung an Postwachstumsfragen diskutiert. Hierbei zeigt sie die Verteilungsmechanismen des deutschen Forschungssystems auf, die insgesamt nur wenig Ressourcen für Forschung im Sinne der sozial-ökologischen Transformation zur Verfügung stellen. Sie plädiert für eine veränderte Ausrichtung weiter Teile der gegenwärtigen Wissenschafts- und Forschungslandschaft in der Hinsicht, dass dem Austausch zwischen Wissenschaft und zivilgesellschaftlichen Akteuren mehr Relevanz und entsprechende Foren und Mittel zugestanden werden. Damit würde zumindest die Chance steigen, dass Postwachstumsideen mehr Beachtung finden. Im abschließenden Beitrag thematisieren schließlich Jürgen Kopfmüller, Linda Nierling, André Reichel und Marius Albiez das Verhältnis zwischen Postwachstumsund Nachhaltigkeitsdebatte, das zwar a priori als spannungsreich erscheint, in dem jedoch auch konzeptionelle und empirische Gemeinsamkeiten gefunden werden können. Auch vor dem Hintergrund der Diskussionen des Workshops plädieren sie für eine differenziertere Sicht auf die Dinge, in der beiden Debatten und entsprechenden Forschungsarbeiten die ihnen gebührende Bedeutung zukommt, um einen Beitrag zum Erreichen gemeinsamer Ziele leisten zu können.

Die Diskussionen während des Workshops aber auch die Beiträge in diesem Schwerpunkt zeigen zum einen, dass der Postwachstumsbegriff und entsprechende Konzepte sehr divers und heterogen sind und damit zugleich Anlass zu vielfältigen Kontroversen bieten. Zum anderen bergen gerade diese Eigenschaften die Chance, Postwachstumsideen in konstruktiven Kontroversen als Bereicherung bestehender Debatten 
zu verstehen, beispielsweise dadurch, dass dort in Vergessenheit geratene (möglicherweise radikale/ungewöhnliche) Ideen wiederentdeckt und auch wiederbelebt werden. Eine wesentliche Voraussetzung für eine fruchtbare Auseinandersetzung ist allerdings, dass sich die Postwachstumsdebatten und -communities ebenfalls öffnen und dabei Anschlüsse an Debatten in bestimmten Themenfeldern (z. B. Mobilität oder technische Innovationen) oder in konzeptionellen Bereichen wie der sozial-ökologischen Forschung zulassen.

\section{Literatur}

Daly, H.E.; Farley, J., 2003: Ecological Economics: Principles and Application. Washington

Heinberg, R., 2011: The End of Growth: Adapting to Our New Economic Reality. Gabriola Island, BC

Jackson, T., 2009: Prosperity without Growth: Economics of a Finite Planet. London

Latouche, S., 2004: Degrowth Economics. Le Monde Diplomatique. November 2004, S. 15

Latouche, S., 2010: Degrowth. In: Journal of Cleaner Production 18/6 (2010); S. 519-522

Paech, N., 2009: Postwachstumsökonomie - Ein Vademecum. In: Zeitschrift für Sozialökonomie 46/160 161 (2009), S. 28-31

Speth, J.G., 2009: Doing Business in a Postgrowth Society. In: Harvard Business Review 87/9 (2009), S. 18-19

\section{Kontakt}

Jürgen Kopfmüller

E-Mail: juergen.kopfmueller@kit.edu

Dr. Linda Nierling

E-Mail: linda.nierling@kit.edu

Institut für Technikfolgenabschätzung und

Systemanalyse (ITAS)

Karlsruher Institut für Technologie (KIT)

Karlstraße 11, 76133 Karlsruhe

Prof. Dr. André Reichel

Karlshochschule International University

Karlstraße 36-38, 76133 Karlsruhe

E-Mail: areichel@karlshochschule.de

\section{Degrowth und Postwachstum - Reflexionen zu Konzeptvielfalt und Nachhaltigkeitsbezügen}

\author{
von Stephan Lorenz, Friedrich-Schiller- \\ Universität Jena*
}

Mit den Themen „Degrowth" und „Postwachstum" wurde in den vergangenen Jahren Wachstumskritik wieder auf die politische und wissenschaftliche Agenda gehoben. Die Vielfältigkeit der Debatten zu systematisieren, gelingt bislang nur unzureichend. Gründe dafür werden in diesem Beitrag diskutiert. Zum einen bleiben die Begriffsbestimmungen von Wachstum bzw. Wachstumskritik und der Ideen von Degrowth bzw. Postwachstum häufig unausgeführt oder als Negativbegriffe (De-I Post-) unkonkret. Das kann den Vorzug bieten, viele Ideen unter einem Dach zu versammeln, erweist sich aber als problematisch im Hinblick auf Entwürfe von Alternativoptionen. Zum anderen fehlt es bisher, auch aufgrund der verbreiteten Ablehnung des Paradigmas nachhaltiger Entwicklung in diesen Debatten, an einem tragfähigen gesellschaftlichen Entwicklungsverständnis. Abschließend werden Vorschläge für eine allgemeine Bestimmung von Wachstumskritik und alternative soziologische Entwicklungskonzepte benannt.

With discussions about de-growth and post-growth the criticism of growth has re-emerged on the political and scientific agenda over the last years. To systematise the diversity of the debates has hardly been attained so far. The article will discuss reasons for this. Firstly, the terms and concepts of growth and growth criticism as well as the ideas of de-growth/post-growth are often not determined or stay vague because of their negative connotations (de-/post-). That may offer the advantage of being able to assemble many ideas under one roof. However, it proves to be problematic with regard to alternative options for society. Secondly, solid conceptions of development are missing so far, not at least because of a widespread rejection of the sustainable development paradigm in these debates. The article suggests a general determination of growth criticism as well as alternative sociological conceptions of development. 